\title{
Meta-Heuristic Procedure for Enhanced Spectrum Fragmentation Assessment in Elastic Optical Networks
}

\author{
Clayton J. N. Lira, Raul C. Almeida Jr., Daniel A. R. Chaves, Helio Waldman and Karcius D. R. Assis
}

\begin{abstract}
This work proposes the use of a procedure for improving the loss of capacity assessment of heterogeneous bandwidth requests in the min slot-continuity capacity loss (MSCL) spectrum allocation algorithm. The particle swarm optimization (PSO) method is used to determine the best relationship between the number of requested slots and the amount of contiguous free slots in the requested and interfering paths. We also incorporate in the MSCL the influence of the number of hops of a route in its capacity evaluation. Considerable reductions in the path request blocking probability are achieved when the technique is applied, particularly when the number of hops is included to the evaluation.
\end{abstract}

Index Terms-Elastic optical network, particle swarm optimization.

\section{INTRODUCTION}

The growing demand for bandwidth experienced by transport networks in recent years has been driven by the improved access to the Internet and by new video distribution applications, social networks, data centers services, among others. Optical communication networks have been the preferred technology to support this increasing necessity for bandwidth. These networks have used, in the last decades, the concept of wavelength division multiplexing (WDM), which causes several fixed-bandwidth connections that can together boost the link capacity to few Tbps [1]. However, with the advent of heterogeneous and variable-traffic demands, the requirement of using fixed-bandwidth channels have made the WDM technology not efficient [1].

Elastic optical networks (EONs) have been proposed as a promising candidate for appropriately supporting high and adaptive transmission rates in optical transport networks. In such networks, the optical frequency spectrum is divided into narrow-width slots and any sequence of contiguous, available slots on all the links of a chosen route may be assigned to the connection. This is performed by the routing and spectrum assignment (RSA) problem. As a consequence, EONs can provide variable-bandwidth optical paths that can properly

Clayton J. N. Lira and R. C. Almeida Jr. are with the Federal University of Pernambuco (UFPE), Campus Recife, Pernambuco, Brazil.

D. A. R. Chaves is with Polytechnic School of Pernambuco (Poli), University of Pernambuco (UPE), Campus Recife, Pernambuco, Brazil.

Helio Waldman is with University of Campinas (UNICAMP), Campinas, SP, Brazil.

Karcius Assis is with Federal University of Bahia (UFBA), Salvador, BA, Brazil.

The authors would like to thank the financial support of Capes, Facepe, Fapesp (Proc. 2015/24341-7) and UFPE (Proc.23076.024114/2018-02).

Digital Object Identifier: 10.14209/jcis.2020.33 adapt to heterogeneous demands [2]. Although EONs present a more efficient utilization of the spectrum when compared to its WDM counterpart [1], dynamically setting up and tearing down connections can generate bandwidth fragmentation [3], defined as the condition where available slots become isolated from each other by being misaligned along the routing path or not contiguous in the spectrum domain [4]-[7]. Spectrum fragmentation suppresses resource utilization, which enhances call blocking in the network. Mitigation of spectrum fragmentation has to be accounted by an efficient RSA algorithm.

Many authors have proposed methods to deal with the fragmentation problem. Some of them, as in [5], proposed ILP (integer linear programming) for static traffic networks. The authors proposed ordering the connection demands, serving first the ones that require the highest number of subcarries, and giving priority to the demands whose shortest path utilizes the largest number of links.

In [8] the authors proposed fragmentation- and alignmentaware algorithms to alleviate the fragmentation due to service provisioning. The fragmentation-aware RMSA calculates the number of divisions in spectrum blocks caused by all RMSA candidates and chooses the one with the minimum number of divisions. The algorithms presented in [9] divided the RSA problem in several steps, first evaluating the network capabillity based on the distribution of traffic and the incoming traffic bandwidth, then modifying routing schemes to achieve low computational complexity. They also proposed an optimized weighted spectrum allocation algorithm based on the trade-off between minimizing the spectrum slot sequence number and maximizing the anticipated network capability.

The main aim of this paper is to propose two heuristic methods for best assessing how the allocation of available slots upon the establishment of a connection impacts the capacity of establishing upcoming heterogeneous-bandwidth requests using the very efficient spectrum assignment mechanism known as min slot-continuity capacity loss (MSCL) [10]. Particle swarm optimization (PSO) technique [14] is used to perform this assessment by finding the most efficient relation between spectrum fragmentation, number of requested slots and/or route length, with the objective of reducing overall network path request blocking probability. This paper provides an important opportunity to promote further understanding of spectrum allocation in EONs.

In Section II, the aspects of the MSCL algorithm are discussed. Section III presents the proposed MSCL refinement. Section IV is concerned with a new methodology used for this 
study by PSO algorithm. Section V presents the findings of the research and analyses the results of simulations. Section VI draws upon the entire paper, tying up the results and includes a discussion of the implication of the findings to future research.

\section{MSCL ALGORITHM}

MSCL is a spectrum assignment algorithm designed for EONs [10]. It searches for the set of contiguous slots in the requested route that leads to the lowest loss of capacity of forthcoming heterogeneous-bandwidth demands, with the aim of reducing the overall network path request blocking probability. The loss of capacity resulting from the connection establishment is determined by considering the loss of capacity in both the requested route and interfering routes. Interfering routes are those that share at least one link with the route selected for the request. MSCL is based on the fact that different spectral ranges that can be used to allocate a request may impact differently the capacity of establishing upcoming heterogeneous-bandwidth connections. Therefore, if such impacts are properly quantified, one can select the spectral range that preserves the capacity of upcoming heterogeneousbandwidth connections as much as possible, resulting in a higher potential of establishing upcoming connections. A brief description of the concepts behind the MSCL spectrum assignment technique is provided below.

Let us assume a network in which requests may demand different amounts of spectrum slots, with all possible numbers of demanded slots being taken from a set $\mathcal{N}$, thus characterizing classes of requests for service.

A spectrum void is defined as a set of contiguous slots that are free in all links of a route. Let the $m$-th spectrum void of a route $r$ be represented as $h_{m}^{<r>}$, where $\mid h_{m}^{<r>\mid}$ is the number of contiguous slots in such void. Suppose a request demanding $i$ slots has just arrived to the network and the route $r$ has been assigned to it. The SA (Spectrum Assignment) algorithm may select any of the voids $h_{m}^{<r>}$ that satisfy $\left|h_{m}^{<r>}\right| \geq i$ (referred to as available voids), and any set of $i$ contiguous slots inside this void.

Note that there might exist several choices for $h_{m}^{<r>}$ and the set of contiguous slots in $h_{m}^{<r>}$ that can be used to establish the current incoming $i$-slot demand. These possible choices may differently affect the network capacity of accommodating requests. We define, respectively, states $\psi$ and $\psi^{\prime}$ as the network state immediately prior and after a possible spectrum allocation of the incoming $i$-slot request. Thus, the network loss of capacity after accommodating any $n$-slot demand $(n \in \mathcal{N})$ may be defined as follow [10]:

$$
C^{<r>}(n)=\sum_{p \in I_{r}} S^{<p>}(\psi, n)-S^{<p>}\left(\psi^{\prime}, n\right),
$$

where the capacity $S^{<p>}(\psi, n)$ is defined as the number of possible allocations for setting up $n$-slot requests on the available (i.e. free) slots of a route $p$ under network state $\psi$ and $I_{r}$ is the set formed by the route $r$ under analysis and all routes that interfere with it.

The total loss of capacity in the network must be calculated over the entire set of possible heterogeneous-bandwidth traffic demands, which is given by:

$$
C_{\mathrm{N}}^{<r>}=\sum_{n \in \mathcal{N}} C^{<r>}(n),
$$

The MSCL SA algorithm checks all available $h_{m}^{<r>}$ and all possible alternatives of allocating the current $i$-slot demand in $h_{m}^{<r>}$, and selects the possibility that returns the lowest value for $C_{\mathrm{N}}^{<r>}$.

Notice that there are different possibilities of defining $S^{<p>}(\psi, n)$, each of which results in a different quantification of the spectrum availability relevance to heterogeneousbandwidth requests. A judicious choice was to define $S^{<p>}(\psi, n)$ as the number of forms of allocating a $n$-slot request in a path [10], henceforward referred to as original MSCL. The number of ways how a $n$-slot request may be allocated in a void $h_{m}^{<p>}$ is given by $\left|h_{m}^{<p>}\right|-n+1$ whenever $\left|h_{m}^{<p>}\right| \geq n$ and 0 otherwise. Therefore, if $M$ is the number of spectrum voids in a route $p, S^{<p>}(\psi, n)$ can be evaluated using [10]:

$$
S^{<p>}(\psi, n)=\sum_{m=1}^{M} \max \left(0,\left|h_{m}^{<p>}\right|-n+1\right),
$$

\section{MSCL REFINEMENT}

The original definition of $S^{<p>}(\psi, n)$ as the number of ways of allocating a $n$-slot request in a route $p$, as stated in Eq. (3), together with the loss of capacity quantification provided by Eqs. (1) and (2) provide a convenient form of quantifying the importance of spectrum voids' suppression under heterogeneous-bandwidth requests. This is confirmed by the convincing reductions in the network path request blocking probability when MSCL is compared to the First-Fit spectrum assignment algorithm, as discussed in [10]. In spite of its confirmed efficiency, the quantification of the loss of capacity as originally proposed in the original MSCL technique is based on a heuristic procedure and therefore may be subject to further improvements.

Let us think in the relation between an arbitrary void $h_{m}^{<p>}$ in a route $p$ and a heterogeneous-bandwidth connection request for $n$ slots as a matrix $\mathcal{M}$, where column $j$ represents a spectrum void of size $j$ and line $i$ a request for $i$ slots. Therefore, $\mathcal{M}(i, j)$ may be used to quantify the importance that a $j$-size spectrum void represents to an $i$-slot request. By doing this, the capacity $S$ to accommodate a $n$-slot request may be evaluated as:

$$
S^{<p>}(\psi, n)=\sum_{m=1}^{M} \mathcal{M}\left(n,\left|h_{m}^{<p>}\right|\right),
$$

If we set the elements of this matrix as the number of ways how an $i$-slot request may be assigned to a $j$-size void, which, using Eq. (3), is given by $j-i+1$ whenever $j \geq i$ and 0 otherwise, matrix $\mathcal{M}$ may be used as an alternative way to calculate $S^{<p>}(\psi, n)$ in the original MSCL. However, as stated before, the number of ways is a heuristic proposition and therefore may not describe the most efficient manner of relating the importance of a spectrum void to forthcoming heterogeneous-bandwidth demands. It is possible, then, that more efficient spectrum assignments may be achieved by the 
use of alternative values for quantifying the importance function $\mathcal{M}(i, j)$ of a $j$-size spectrum void over an $i$-slot request. To find optimized values to fill the matrix, we propose to use the particle swarm optimization technique, which searches for adequate values to $\mathcal{M}(i, j)$ elements that achieve the lowest possible overall network path request blocking probability.

Other features may be included to contemplate not only the importance of a void to connections with different number of slots, as proposed in the original MSCL, but also the difficulty of allocating connections with different number of slots and hops, once the difficulty of finding available resources in the network also increases with the number of hops in the route $p$ chosen by the routing algorithm. Therefore, we propose in this paper a modification to the original MSCL structure by including the route' number of hops in the network loss of capacity assessment. This is performed with the use of a matrix $\mathcal{M}(i, j, k)$, where the extra $k$ sub index stands for the number of hops in the route. Therefore, now, different-size routes shall more appropriately quantify the capacity of available voids to its different heterogeneous-bandwidth requests. By doing this, we can evaluate the capacity of a route $p$, with $|p|$ hops, in accommodating a $n$-slot request as:

$$
S^{<p>}(\psi, n)=\sum_{m=1}^{M} \mathcal{M}\left(n,\left|h_{m}^{<p>}\right|,|p|\right) .
$$

With this strategy, we hope to refine the spectrum fragmentation assessment by including specific characteristics of each network.

The two spectrum assignment alternatives proposed in this paper are stated and described in details below:

- MSCL-W: This strategy, referred to as request-width MSCL optimization, maintains the same procedure proposed by the original MSCL of inferring the capacity of heterogeneous-bandwidth requests based on the void size and number of requested slots. However, MSCL-W searches for a more efficient form of quantifying such capacity, instead of heuristically quantifying it through the number of forms, as in Eq. (3). Therefore, the matrix $\mathcal{M}(i, j)$ and Eq. (4) are used by MSCL-W and an optimization process is used to adjust the elements of $\mathcal{M}(i, j)$.

- MSCL-WL: This approach, referred to as request-widthand-length MSCL optimization, extends the limited perception of quantifying the impact of spectrum void suppression based only on the requested bandwidth of the demands, as in the original MSCL and MSCL-W, by including the awareness that different-length requests must also experience different spectrum-void suppression penality. Therefore, spectrum void suppression can be differently quantified by connections with the same bandwidth, but different lengths, and vice-versa. In this case, the route-capacity evaluation is performed using the matrix $\mathcal{M}(i, j, k)$ and Eq. (5), and the same optimization process as in MSCL-W is used to adjust the elements of $\mathcal{M}(i, j, k)$.

There are two phases in order to implement our proposed optimized MSCL procedure: the design and operational phases. These phases are defined as:

- The design phase occurs prior to the network operation. The PSO is used to find optimized values either for $\mathcal{M}(i, j)$ or $\mathcal{M}(i, j, k)$. The PSO is used to optimize the following problem: given the network topology, a large set of connection requests generated under a Poisson stochastic process, a routing algorithm and the SA MSCL algorithm (using either equations 1,2 and 4 or 1,2 and 5 ), return the values for $\mathcal{M}(i, j)$ (or $\mathcal{M}(i, j, k)$ ) elements that achieve the lowest network path request blocking probability. The blocking probability is evaluated using a network simulator which solves the SA using either MSCL-W or MSCL-WL.

- The operational phase uses the optimized values for $\mathcal{M}(i, j)$ (or $\mathcal{M}(i, j, k)$ ) elements along the MSCL SA algorithm (using either equations 1,2 and 4 or 1,2 and 5) to perform the SA for each incoming connection request.

\section{Particle Swarm Optimization (PSO) Technique}

The PSO algorithm is used to find optimized values for the matrices that are used for evaluating the capacity of the two proposed heuristics, as previously stated. The PSO algorithm is a meta-heuristic iterative optimization algorithm that uses a swarm intelligence approach. The algorithm iteratively updates the position and velocity of a set of particles in order to minimize an objective function. The position of a particle in the space represents a possible solution for the problem. In our proposal, the particle position represents the elements' value of either $\mathcal{M}(i, j)$ (MSCL-W) or $\mathcal{M}(i, j, k)$ (MSCL-WL), whereas the objective function is the overall network path request blocking probability returned by network simulations. In the MSCL-W approach, each particle is composed by the elements of an $N x M$ matrix, arranged in a vectorized form. The quantity $N$ represents the number of different $n$-slots requests allowed in the network and $M$ is the maximum number of slots in a spectrum void. A similar representation is used for MSCL-WL, but now there are $K$ instances of $N \times M$ matrices, in which $K$ is the maximum hop count for any route in the investigated topology.

The PSO particles communication was implemented with the local best model (Lbest) in a ring topology. In this model, each particle communicates with $c$ neighbors. In this work, we set $c=2$, as adopted in several works in the literature [12], [13]. In addition, it was used 30 particles and 120 iterations as stop criterion. The particle velocity update equation used and the complete PSO pseudo code assumed are the same as described in [12].

\section{Simulation Results and Discussions}

The performance of the two proposed methods for improving the MSCL methodology is verified through simulations. The simulator was developed and tested by the UFPE's research group and several other works were published using the simulator's basic structure [11], [15], [16]. Dijkstra's shortestpath algorithm was used as routing scheme. The dynamic network scenario adopted throughout this paper considers incoming heterogeneous-bandwidth requests arriving to the 


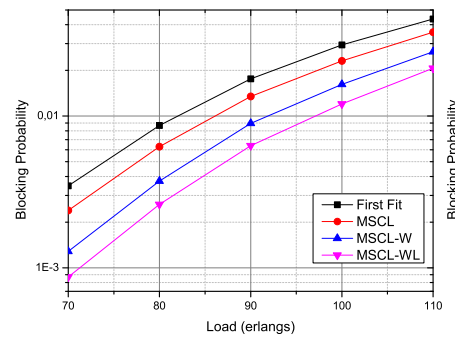

(a) NSFNet topology

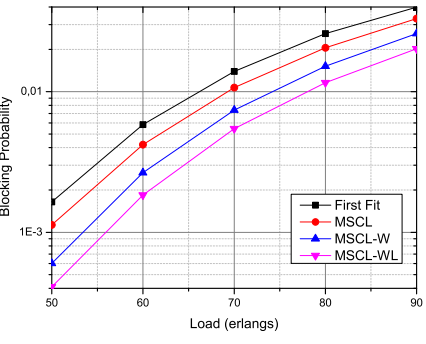

(b) Finland topology
Fig. 1: Blokcing probability as a function of load obtained by the FF, MSCL, MSCL-W and MSCL-WL algorithms

system following a Poisson process and their holding time randomly generated following an Exponential distribution. The source-destination node pairs are randomly chosen with equal probability.

Fig. 1 shows the path request blocking probability versus the network offered load (in erlang) when either First-Fit (FF), original MSCL [10] or one of the proposed MSCL extensions (MSCL-W and MSCL-WL) are used as spectrum assignment heuristics. We have compared the performance of such SA algorithms for the NSFNet (Fig. 1 (a) and Finland (Fig. 1 (b)) topologies, which are composed of 14 nodes and 42 links and 12 nodes and 38 links, respectively [11]. A total of 64 slots per link is used, each with a width of $12.5 \mathrm{GHz}$, and traffic requests varying uniformly between $2,3,4,5$, and 6 slots.

The PSO iterative processes optimization for both MSCLW and MSCL-WL was performed considering the load value of 110 erlangs for the NSFNet topology and 90 erlangs for the Finland topology. Then, the optimized solution (i.e. the values stored in the elements of matrix $\mathcal{M}$ ) obtained for such traffic load intensity is used to obtain the network blocking probabilities values of the proposed extended MSCL heuristics.

As already stated in [10], the use of the original MSCL heuristic provides noticeable reduction to the path request blocking probability when compared to the FF spectrum assignment. However, notice that considerable further mitigation on the path request blocking probability is achieved when either MSCL-W or MSCL-WL are used. The use of the proposed MSCL-W or MSCL-WL results in lower blocking probability values than the FF and original MSCL algorithms in the entire investigated load range in both investigated topologies. The error bars in the blocking probability outcomes are for a confidence interval of $95 \%$, and are too small to be perceived in the graphs. Moreover, one can note that the MSCL-WL ouperforms MSCL-W in the investigated topologies and load ranges.

This fact is due to the inclusion of the length of the routes in the optimization process, since the difficulty of finding resources on the route depends on it (routes with many hops have more interfering routes, which use their resources in intermediate nodes and links). The inclusion of the number of hops as variable in the process makes possible to find more suitable values for filling out each matrix and, consequently, for calculating the allocation cost in each route.
We highlight the importance of the MSCL-W and MSCLWL results, since they are derived from an optimization process of an algorithm that is already considered efficient in the literature for spectrum assignment in elastic optical networks. This indicates that the proposed strategies can better quantify how severe is the effect of a fragmented spectrum to requests with different number of requested slots. In addition, when the number of hops is combined to the number of requested slots, a more rational assessment is achieved.

Table I shows the Average percentage path request blocking probability reduction of MSCL when compared to the FF spectrum assignment, and MSCL-W and MSCL-WL when compared to MSCL in the NSFNet and Finland topologies. The results show the average reduction value under all considered loads. We have calculated the percentage reduction by subtracting the blocking probability of the heuristics and dividing this value by the highest blocking probability. Comparing the simulated topologies, it is observed that the best average gain is obtained for NSFNet, with path request blocking probability reductions of about $53 \%$, whereas an average reduction of $50 \%$ was obtained for Finland topology.

TABLE I: Average percentual path request blocking probability reduction.

\begin{tabular}{lll}
\hline Algorithms & NSFNet & Finland \\
\hline FF/MSCL & $24 \%$ & $24 \%$ \\
MSCL/MSCL-W & $35 \%$ & $32 \%$ \\
MSCL/MSCL-WL & $53 \%$ & $50 \%$ \\
\hline
\end{tabular}

\section{CONCLUSIONS}

In this work, we propose two heuristics to solve the problem of spectrum assignment in elastic optical networks: MSCL-W and MSCL-WL. The heuristics are designed to improve the evaluation of the network loss of capacity of heterogeneousbandwidth demands as originally proposed by the MSCL. While MSCL-W keeps the structure of the original MSCL and adequately adjusts the way how the capacity shall be assessed for improved network performance, MSCL-WL adds to the capacity assessment the number of hops in the route under analysis, and optimizes the problem accordingly.

The heuristics' optimization process is performed by the meta-heuristic algorithm from swarm intelligence theory named particle swarm optimization (PSO). We conducted simulation validation of the spectrum assignment proposals on the NSFNet and Finland topologies and the simulation results show that MSCL-W and MSCL-WL achieve an average reduction in the network path request blocking probability that varies between $32 \%$ for MSCL-W algorithm in Finland topology and 53\% for MSCL-WL in NSFNet topology, when both are compared to the original MSCL. There is abundant room for further progress in determining spectrum assignment algorithms used in this paper considering virtualization over a physical EON, $5 \mathrm{G}$ network slicing, and other approaches that use EON technology as a physical substrate.

\section{ACKNOWLEDGMENT}

The authors thank the institutional support of UFPE, UPE, Unicamp and UFBA. 


\section{REFERENCES}

[1] Sahar Talebi, Furqan Alam, Iyad Katib, Mohamed Khamis, Reda Salama, George N. Rouskas, "Spectrum management techniques for elastic optical networks: A survey," Opt. Switching and Netw., Volume 13, Pages 34-48, 2014, ISSN 1573-4277, doi: 10.1016/j.osn.2014.02.003.

[2] M. Jinno, H. Takara, B. Kozicki, Y. Tsukishima, Y. Sone and S. Matsuoka, "Spectrum-efficient and scalable elastic optical path network: architecture, benefits, and enabling technologies," in IEEE Commun. Mag., vol. 47, no. 11, pp. 66-73, Nov. 2009, doi: 10.1109/MCOM.2009.5307468.

[3] B. C. Chatterjee, N. Sarma and E. Oki, "Routing and Spectrum Allocation in Elastic Optical Networks: A Tutorial," in IEEE Commun. Surveys Tuts., vol. 17, no. 3, pp. 1776-1800, 2015, doi: 10.1109/COMST.2015.2431731.

[4] Chatterjee, Bijoy, and Eiji Oki, Elastic Optical Networks: Fundamentals, Design, Control, and Management, CRC Press, 2020. ISBN-10: 1138611719. ISBN-13: 978-1138611719.

[5] K. Christodoulopoulos, I. Tomkos and E. A. Varvarigos, "Elastic Bandwidth Allocation in Flexible OFDM-Based Optical Networks," in J. of Lightw. Technol., vol. 29, no. 9, pp. 1354-1366, 2011, doi: 10.1109/JLT.2011.2125777.

[6] M. Zhang, W. Shi, L. Gong, W. Lu and Z. Zhu, "Bandwidth defragmentation in dynamic elastic optical networks with minimum traffic disruptions," IEEE Int. Conf. on Commun. (ICC), Budapest, 2013, pp. 3894-3898, doi: 10.1109/ICC.2013.6655165.

[7] M. Zhang, C. You, H. Jiang and Z. Zhu, "Dynamic and Adaptive Bandwidth Defragmentation in Spectrum-Sliced Elastic Optical Networks With Time-Varying Traffic", in J. of Lightw. Technol., vol. 32, no. 5, pp. 1014-1023, 2014, doi: 10.1109/JLT.2013.2296781.

[8] Y. Yin, M. Zhang, Z. Zhu and S. J. B. Yoo, "Fragmentation-aware routing, modulation and spectrum assignment algorithms in elastic optical networks," in Opt. Fiber Commun. Conf. and Exposition and the Nat. Fiber Opt. Engineers Conf. (OFC/NFOEC), Anaheim, CA, 2013, pp. 13, doi: 10.1364/OFC.2013.OW3A.5.

[9] X. Chen, J. Li, P. Zhu, R. Tang, Z. Chen and Y. He, "Fragmentationaware routing and spectrum allocation scheme based on distribution of traffic bandwidth in elastic optical networks," in IEEE/OSA J. of Opt. Commun. and Netw., vol. 7, no. 11, pp. 1064-1074, 1 Nov. 2015, doi: 10.1364/JOCN.7.001064.

[10] R. C. Almeida, A. F. Santos, K. D. R. Assis, H. Waldman and J. F. Martins-Filho, "Slot assignment strategy to reduce loss of capacity of contiguous-slot path requests in flexible grid optical networks," in Electron. Lett., vol. 49, no. 5, pp. 359-361, 28 Feb. 2013, doi: 10.1049/el.2012.4247.

[11] C. J. N. de Lira, R. C. Almeida and J. F. Martins-Filho, "On combining Split Spectrum technique with a Slot-Continuity Capacity Loss heuristic in Elastic Optical Networks," in IEEE 18th Int. Conf. on High Performance Switching and Routing (HPSR), Campinas, 2017, pp. 1-6, doi: 10.1109/HPSR.2017.7968687.

[12] Chaves, Daniel Aguiar, Douglas Bastos-Filho, Carmelo Martins Filho, Joaquim, "A methodology to design the link cost functions for impairment aware routing algorithms in optical networks," Photonic Netw. Commun., 22, 133-150, 2011, doi: 10.1007/s11107-011-0314-2.

[13] D. Bratton and J. Kennedy, ”Defining a Standard for Particle Swarm Optimization," in IEEE Swarm Intelligence Symp., Honolulu, HI, 2007, pp. 120-127, 2007, doi: 10.1109/SIS.2007.368035.

[14] Kennedy, James, and Russell Eberhart. "Particle swarm optimization." in Proceedings of ICNN'95-Int. Conf. on Neural Netw., Vol. 4. IEEE, 1995, doi: 10.1109/ICNN.1995.488968.

[15] Correia, B., Almeida Jr, R., Pereira, H., Chaves, D, "Superchannel Multicasting Module Placement Algorithm Applied to Elastic Optical Networks," J. of Commun. and Inf. Syst., 34(1), 131-140, 2019, doi: 10.14209/jcis.2019.14.

[16] B. V. A. Correia, R. C. Almeida, D. A. R. Chaves and H. A. Pereira, "Optical Inverse Multiplexing Technique Applied to Elastic Optical Networks," in 21st Int. Conf. on Transparent Opt. Netw. (ICTON), Angers, France, 2019, pp. 1-4, doi: 10.1109/ICTON.2019.8840471. 